www.jmscr.igmpublication.org

Impact Factor 5.84

Index Copernicus Value: 71.58

ISSN (e)-2347-176x ISSN (p) 2455-0450

crossref DOI:_https://dx.doi.org/10.18535/jmscr/v6i1.82

\author{
Journal Of Medical Science And Clinical Research \\ IGM Publication \\ An official Publication of IGM Publication
}

\title{
Critical Appraisal of Two Techniques of Manual Construction of Tissue Microarray
}

\author{
Authors \\ Aakanksha Singh ${ }^{1}$, Usha Rani Singh ${ }^{2}$, Sonal Sharma ${ }^{3}$, Navneet Kaur ${ }^{4}$ \\ ${ }^{1}$ MD (Pathology), MBBS, UCMS \& GTB Hospital \\ ${ }^{2}$ Director Professor, Department of Pathology, UCMS \& GTB Hospital \\ ${ }^{3}$ Director Professor, Department of Pathology, UCMS \& GTB Hospital \\ ${ }^{4}$ Director Professor, Department of Surgery, UCMS \& GTB Hospital
}

\begin{abstract}
Introduction: Tissue microarray (TMA) is a technology, which has come up in the recent years and has application in carrying out high throughput immunohistochemical and molecular analysis. The widespread use of this method in developing countries like India has been limited by the high cost of the automated tissue arrayers. The advantages of this technology has led researchers to find suitable and lesser expensive manual alternatives to creating such microarrays.

In this study, we used two such manual methods, described by Kononenet al ${ }^{l}$ using prefabricated recipient blocks and the other by Chen et al ${ }^{2}$ without the use of prefabricated recipient blocks, to manually create such microarrays. The aim of this study was to assess the efficacy of either or both the methods and also comparing the outcome differences in the preparation of standard $H \& E$ slides of cases of carcinoma breast. The idea was to propose the use of one technique over the other for routine immunohistochemistry in our set-up.

Materials and Methods: 65 cases of histologically confirmed carcinoma breast were included in the study. Microarrays were constructed in a $5 X 3$ grid using two manual methods, described by Kononenet al $l^{1}$ and the other by Chen et al ${ }^{2}$. Immunohistochemistry for HER-2, ER, PR and CK 5/6 were done on the TMA sections and compared with routine sections.

Results: Both the methods for construction of TMA manually were found to be easy and economical for our setup. The use of tissue microarrays decreased the total staining time and also decreased the total amount of reagents used, decreasing the total expenditure. The Chen technique of TMA construction proved to be an easier technique to learn than the first.

Problems associated with the Kononen technique $e^{l}$ were significant core loss and core mal-alignment, cores not being flushed to surface, broken cores and block cracking. Problems associated with the Chen technique ${ }^{2}$ core tipping and cumbersome removal of adhesive tape.

There were averagely 20 sections obtained with almost all cores with the technique suggested by Chen et al, ${ }^{2}$ on the other hand there were hardly any sections with all the cores with the technique suggested by Kononen et al. ${ }^{1}$ This could entail loss of assessment of one or the other tumor specimen or too few slides if all the cores were to be studied together.

Discussion: Therefore, if few slides are necessary, fewer specimens (20-30) have to be studied and no precise arrangement is necessary then Kononen technique ${ }^{l}$ is recommended. And if, many slides are necessary, there are few specimens (30-50) and precise arrangement is necessary, then Chen technique: TMA with double sided adhesive tape, is recommended. 2,3

Keywords: Tissue Microarray, Manual, Immunohistochemistry.
\end{abstract}




\section{Introduction}

Tissue microarrays are composite paraffin blocks constructed by extracting cylindrical tissue core "biopsies" from different paraffin donor blocks and re-embedding these into a single recipient (microarray) block at defined array coordinates. ${ }^{4}$ TMAs have become a mainstay in preclinical and translational research, especially for the development of biomarker assays for characterization of disease. They allow analysis of extremely small amounts of tissue, thus preserving valuable tissue blocks. At the same time, they dramatically increase the efficiency and costeffectiveness of performing tissue-based studies, ${ }^{5,6}$ by enabling the examination of $10 \mathrm{~s}$ to 100 s of different patient samples on the same slide. This approach also leads to greater reproducibility and comparability of patient samples since all samples are exposed to identical processing conditions. $^{7}$

Placement of multiple tissues from multiple patients/organs in a single block/slide were described first in 1986 by Hector Battifora who termed it the 'sausage' tissue block, ${ }^{8,9}$ but the process was laborious and time consuming. The use of TMAs did not become popular until Kononen et al. developed a mechanized version of TMA construction ${ }^{14}$ since then many techniques for construction of tissue microarray have come up.

Today, automated tissue microarray machines are so expensive that even the tertiary care hospitals do not routinely use it. In such a scenario manually constructed tissue microarrays, bridge the gap between inexpensive outreach to peripheral centers and uniform, standardized high throughput tissue analysis.

Most of these techniques of construction of TMA are centered around, either with the use of prefabricated paraffin blocks or without it. Any essential difference between the two methods, in the outcome of the array and in the objective ease of preparation, intends scientific enquiry. An efficient and cost effective manual tissue microarray assessed on objective parameters might prove a technological asset for research centers and hospitals with high throughput analysis burden.

The present study is an effort to study the utility of creating manual tissue microarrays using simple, cheap and easily available equipment and studying HER-2 and hormone receptor expression by IHC in breast cancer patients in these microarrays, thereby maximizing the benefits and reducing the overall expenditure, without affecting the precision and accuracy of the results.

\section{Materials and Methods}

The study was conducted in the Department of Pathology at UCMS \& GTB Hospital, Delhi, for 15 months.

Type of Study: Descriptive, consecutive, cross sectional study.

Consent and Ethics: Written informed consent and institutional ethical clearance was obtained.

Sample Size: 65 cases of carcinoma breast were taken for the study.

Inclusion Criteria: Tissues from confirmed carcinoma breast biopsy samples and mastectomy cases were included in the study.

\section{Exclusion Criteria}

- Non availability of representative tissue of the tumor.

- Poor tissue processing.

\section{Materials}

1. Donor blocks (grossed from tumor proper of specimen).

2. Bone marrow trephine biopsy needles, sizes $14 \mathrm{G}$ and $16 \mathrm{G}$.

3. Paraffin Wax (Merc corporation).

4. Micro slides (PIC-1, Blue star, polar industrial corporation, Mumbai).

5. Hot air Oven (Tanco Universal oven PLT $125 \mathrm{~A})$.

6. Stainless steel molds, $6 \mathrm{~mm}$ (Yorco).

7. Tissue cassettes.

8. Floatation water bath (Electric tissue float bath- Yorco).

9. Accu Edge blades (Leica 819). 
10. Pilot Pen ultrafine point (Register Office Supply).

11. Semi automatic rotary microtome (Micron HM 340E).

12. Embedding station (Micron EC 350-1).

13. Double sided tape (register office supply).

14. Used X ray sheet.

15. Standard stationary scissors.

16. A pair of small stainless steel forceps

17. Felt pen

\section{Sample Collection}

Specimens signed out as invasive ductal carcinomas/ invasive carcinomas were re-grossed for tumor areas. Protein expression of ER, PR, HER2 and CK 5\&6 status were done on these formalin fixed breast tissues.

\section{Tissue Selection and Donor Block Preparation}

1. The tissue of choice was identified using a standard tissue section on a glass slide stained with hematoxylin and eosin (H\&E).

2. The area to be sampled was circled directly on the glass slide, which usually represented a region corresponding to a specific pathological diagnosis ("tissue diagnosis- breast carcinoma") or with a Pilot Pen (or similar xylene-free pen). Each separate region (tissue diagnosis) was assigned its own number or letter, such that it could be uniquely identified given the case number, block designation, and tissue diagnosis designation. The circled H\&E glass slide was overlayed on the block and the area of interest and the corresponding region on the block was identified and circled with a laboratory marker pen. (Figure A)

\section{Construction of the Tissue Microarray: Tissue} microarrays in this study were constructed manually using the two methods described by Kononen et $\mathrm{al}^{1}$ (using prefabricated paraffin recipient block) and Chen et $\mathrm{al}^{2}$ (without the use of prefabricated paraffin recipient block). Especially cut bone marrow trephine biopsy needles were used. Cores were taken from the donor tissue blocks from the area selected after studying the haematoxylin\& eosin stained sections. The gauge of the needles used were so adjusted that the internal diameter of $14 \mathrm{G}$ fitted the outer diameter of $16 \mathrm{G}$. The core diameter was standardized to $1.5 \mathrm{~mm}$. Arrays of $5 \mathrm{X} 3$ were constructed with a pointer core embedded on one corner for easy orientation. A positive control was selected for pointer core.

\section{Equipment Setup and Method for TMA Construction as Described by Kononen et al ${ }^{\mathbf{1}}$}

1. Preparation of recipient block - The wax was melted at $56-59^{\circ} \mathrm{C}$ and then poured into a deep mold. A standard tissue cassette is placed on top.

2. The block was allowed to chill completely at room temperature. After cooling, the cassette and mold were separated.

3. Each donor block with corresponding $\mathrm{H} \& \mathrm{E}$ section slide were arranged in the order by which they were to be used as per the microarray map design.

4. A pair of bone marrow biopsy needles of $14 \mathrm{G}$ and $16 \mathrm{G}$ were used. The larger bore (14G) was used for extracting tissue from the donor block and the smaller bore(16G) was used for making a hole in the recipient block. (Figure B)

5. The recipient block was placed on a flat levelled surface.

6. To ensure the alignment of the punches, the recipient punches were to be drilled into position marks inscribed on the paraffin.

7. The depth of the punches over the block could be controlled by adjusting the guard of the bone marrow biopsy needle and gently pushing down bone marrow biopsy needle up till the limit of the guard (of the desired depth) was attained.

8. The biopsy needle (16G) was placed on the recipient block over the position inscribed. Pressure was applied to the top of the turret to bring down the needle. A squeezing motion was used to push the biopsy needle into the paraffin. The arm of the punch was rotated to the left and then back to the right, while maintaining pressure 
on the turret top. This rotating motion helped free the core from the recipient paraffin block.

9. The stylus was pressed down to eject the paraffin core from the punch and its length was examined for assessment of depth.

10. Then, the $14 \mathrm{G}$ biopsy needle was placed over the donor block under the area of interest circled. The punch was taken from the inscribed sample area by rotatory movement (as described in step 8) of biopsy needle and the paraffin core from the recipient block was removed, leaving a hole behind. (Figure C\&D)

11. The donor core was inserted into the recipient hole by bringing the needle down until the lower punch surface was directly over the hole that was just created.

12. Gentle pressure was maintained on the needle, slowly pushing down the stylus, guiding the core into the hole while expelling the tissue from the punch. This sample would have the coordinates 01, 01 from the map.

13. Similar procedure was continued across the row until the last core had been placed.

14. The same procedure was repeated in the subsequent rows until the block was complete.(Figure E)

\section{Equipment Setup and Method for TMA Construction as Described by Chen et al. ${ }^{2}$} 1. A bone marrow biopsy needle of $14 \mathrm{G}$ was used for punching cores from the donor blocks.

2. A piece of double-sided adhesive tape was cut exactly according to the size of the embedding mold to provide a uniform cutting surface.

3. The top surface was exposed to receive the tissue cores and the bottom was stuck to a used X ray sheet cut exactly to the shape of the mold for easy detachment of the block later from the mold.

4. The sites for attachment were marked with the help of a ruler and a felt pen, for better core alignment.

5. The area to be cored was marked on the $\mathrm{H}$ and E-stained sections (as described earlier) and then on the block by superimposing the marked slide on it.
6. The bone marrow biopsy needle was drilled into the donor block at the selected site, taking care that its approach was perpendicular for optimum tissue sampling (as described in step 8 of previous procedure).

7. After pulling out the needle, the tissue core was delivered with help of a stylus and transferred to the tape with the help of forceps (cutting surface facing downwards). (Figure F)

8. The tissue cores were arranged in the pre decided format (according to the tissue microarray map). (Figure G)

9. The array platform with the tissue cores in situ was then transferred to the stainless steel mold, although, the TMA can be constructed with the X-ray sheet in the mold. (Figure $\mathrm{H}$ )

10. Melted paraffin was gently poured in to the mold from the sides, taking care to avoid tipping of the cores. (Figure I)

11. To ensure gradual and uniform setting of the block, the mold was initially cooled at room temperature and then transferred to a refrigerator for $20 \mathrm{~min}$.

12. After the block was evenly set, the adhesive tape was peeled off to expose the cutting surface.

\section{TMA Block Sectioning}

1. On completion of construction, the arrays were placed in an oven at 60 degree Celsius for $10 \mathrm{~min}$ face down on a clean glass slide. This facilitated the adherence of the cores to the walls of the punches in the recipient block and the surrounding wax (by the Kononen method).

2. The slide/block combination was removed from the oven and gentle and even pressure was applied. This evened out any irregularities in the block surface and removed the bulging of the block center, if any, that occurred during array construction.

3. Immediately the TMA blocks were placed onto a block of ice so that it is completely cooled before cutting.

4. The temperature in the water bath was set to 55$60^{\circ} \mathrm{C}$ and the blocks were sectioned at $3-4 \mu \mathrm{m}$. 
5. The slides were dried overnight in a vertical position preferably.

6. After drying slides were placed back to back and put into a stack, wrapped tightly with Parafilm, labelled, and then stored at $-20^{\circ} \mathrm{C}$.

\section{Notes}

1. Donor blocks were placed in a hot air oven to warm up slightly, making them a little softer, less likely to crack, and easier to punch.

2. During construction the block began to bulge up when increased number of cores were put. Incorporating more space between cores minimized this. Greater spacing caused less bulging.

3. It may be necessary to use a magnifier during array construction.

4. It is important to face off the recipient block using a dedicated microtome prior to use. This helps ensure that the block face is smooth and that all the arrays that are made would be in the identical plane. This helps to optimize the number of complete sections that an array block may yield.

5. Preferably use a practice block to test out the proper water bath temperature before cutting the first array block.

6. If the area within the block that is selected contains tissue that is quite thin, it may be advantageous to select an alternate sample. Thin donor blocks may not yield many Tissue Microarray slides. In general, the tissue in the donor block should be at least $1.5 \mathrm{~mm}$ thick, although at times certain types of samples may not contain this much tissue. In this case, one must decide on the relative merits of using a TMA vs simply cutting standard blocks and staining standard slides.

7. We also used deep molds. Care should be exercised to avoid trapping air bubbles in the recipient block.

8. A margin of at least $2.5 \mathrm{~mm}$ of paraffin is recommended around the entire array. This prevents the recipient block from cracking during construction and also during sectioning.
9. After doing several punches the stylus/punch complex may retain some paraffin. Moving the stylus up and down and wiping would remove the paraffin, however xylene had to be used when excess.

10. Do not push the donor punch all the way into its respective hole; let it protrude slightly. It could then be gently pressed in further with a clean glass slide. This ensures that its position is level with the recipient block surface, not in too deep or protruding out.

11. It was possible to cut down the size of a donor core that was too long. Eject the core with the stylus and placed on a clean flat surface. A clean razor blade was used to cut the core to the desired length. The core could then be placed into the recipient block with a pair of forceps. This was easier with the larger size punches.

12. Though it was possible, but extremely cumbersome to remove a wrongly placed core with the donor punch. It left some material behind and also damaged the recipient block. A note of it on the map was made.

13. Cores that were placed too deeply were removed with the smaller size needle (recipient needle). An additional core could then be inserted. However, this process should not be considered routine for several reasons. The hole in the recipient block may become enlarged and some residual tissue may be left behind, also such cores may not blend well with the surrounding paraffin.

14. It is quite important to never construct an array block with complete symmetry, as one can lose orientation. Also, for long term storage, Singh et $\mathrm{al}^{10}$ recommended storing the cut TMA slides covered in a layer of wax.

\section{Immunohistochemistry}

The following panel of antibodies were put:

\section{Estrogen Receptor alpha Antibody (SP1)}

Estrogen receptor alpha antibody by thermo scientific was a rabbit inoculated $\mathrm{IgG}$ unconjugated monoclonal (sp1 clone) antibody from thermo scientific. It was used in 1:100 dilution for immunohistochemistry, with tissue 
culture supernatant acting as storage buffer and $15 \mathrm{mM}$ sodium azide as a preservative. It is stored at 4 degree Celsius.

\section{Progesterone Receptor Antibody (SP2)}

Progesterone Receptor Monoclonal Antibody by Thermo scientific is a rabbit inoculated IgG Class unconjugated Monoclonal Antibody (SP2 clone). It is used in 1:100 dilution for immunohistochemistry with tissue culture supernatant acting as storage buffer and $15 \mathrm{mM}$ sodium azide as preservative. It is stored at 4 degree Celsius.

\section{HER-2 / ErbB2 Antibody (SP3)}

HER-2 / ErbB2 Monoclonal Antibody is a rabbit inoculated $\operatorname{IgG}$ class unconjugated monoclonal (sp3 clone) antibody attaching to the extracellular domain of Her-2/ErB2. It is used in 1:100 dilution for immunohistochemistry, with tissue culture supernatant acting as storage buffer, $15 \mathrm{mM}$ sodium azide as preservative. It is stored at 4 degree Celsius.

\section{Cytokeratin 5 \& 6 Antibody}

Cytokeratin $5 \& 6$ antibody by cell marque is a mouse inoculated unconjugated monoclonal (D5/16B4 clone) antibody of $\mathrm{IgG}$ class with cytoplasmic localization used in 1:50 dilution.

Statistical Analysis: Chi square test was used.

\section{Results}

The aim of the study was to compare the two techniques of manually constructing tissue microarray. The following difficulties were observed by the three operators who constructed TMA by both the methods independently. There was a higher incidence of recipient block cracking observed in the Kononen technique. Broken cores was another difficulty often observed in the Kononen technique. Though subjective, two of the three operators found Kononen technique more cumbersome and the learning curve a bit longer.

Placing the core vertically on the adhesive tape was observed to require a level of practice as far as the Chen technique is concerned. Vertical and symmetrical placement required greater trimming of the donor cores. Core tipping was a recurrent problem exclusively encountered in the second technique. Also two operators found the removal of the adhesive post the formation of TMA block quite cumbersome.

The average time taken by first operator to construct TMA by the Kononen technique (TMA I) was 2 hours 26 mins and TMA by the Chen technique (TMA II) was 1 hours 59 mins. The second operator took about 2 hours 21 mins for constructing TMA I averagely and TMA II about 2 hours and 4 mins averagely. The third operator averagely took about 2 hours 16 mins for constructing TMA I and 2 hours and 19 mins for TMA II. However, the mean time taken to construct TMA I was 2 hours 21 mins and TMA II was 2 hours 7 mins (Figure $\mathrm{O}$ ).

With practice it was observed that time required for construction of each type of TMA by all three operators was reducing. However the slope of fall (i.e. the reduction in the time taken to construct each TMA) was much higher in the Chen technique of TMA construction than the Kononen, hence proving to be an easier technique to learn.

The following parameters were assessed objectively for both the TMA techniques. Core tipping was a problem exclusively seen in the Chen technique. On an average 1.2 cores tipped every 5X3 TMA prepared. In the Kononen technique about 3-4 cores were found mal-aligned in every 5X3 TMA prepared. In the Chen technique about 2 cores averagely were found mal-aligned in every 5X3 TMA prepared.

There were about 2-3 cores averagely missing in every 5X3 TMA prepared by the Kononen technique, however only about $0-1$ cores were missing in every 5X3 TMA prepared by Chen technique. Broken core was a problem inherent to the Kononen technique, averagely 1-2 cores were noted to be broken per 5X3 TMA prepared. Averagely 3-4 cores were not flushed to the surface by the Kononen technique and this problem again was inherent to it.

About 1-2 cores incompletely merged to the surrounding paraffin in the TMA s prepared by 


\section{JMSCR Vol||06||Issue||01||Page 31902-31914||January}

Kononen technique and was seen only in an occasional section of TMA $\mathrm{s}$ prepared by the Chen technique. The average score on a visual analog scale given to sections prepared by Kononen technique was 7.4 and to sections by Chen technique was 7.8.

The average number of sections cut with more than $49 \%$ of cores in Kononen technique were 63 and by Chen technique were 81.8 . The average number of sections cut with more than $80 \%$ of cores in Kononen technique were 33.2 and by Chen technique 59.4. The average number of sections cut with more than $86 \%$ of cores in Kononen technique were 14.6 and by Chen technique 42.6. The average number of sections cut with more than $93 \%$ of cores in Kononen technique were 0.0 and by Chen technique 20.6. (Figure P\&Q)



Figure A: Area of interest being marked on tissue block

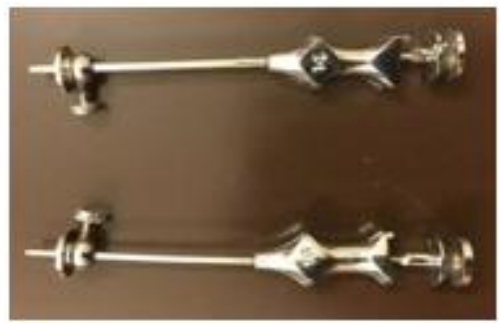

Figure B: Modified Bone marrow biopsy needles used for TMA construction



Figure C: Tissue core being extracted by rotary movement

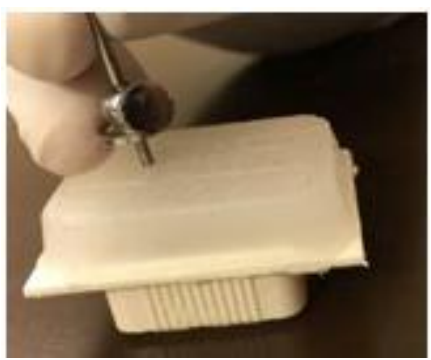

Figure D: Tissue core being inserted into the paraffin recipient as suggested by Kononen

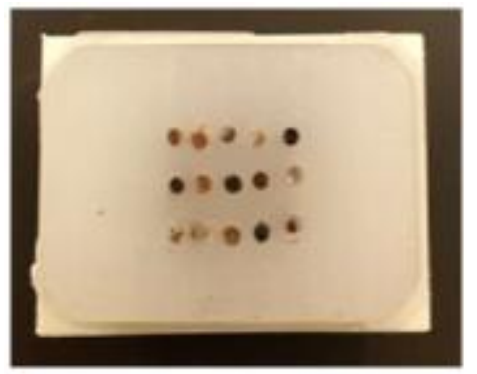

Figure E: Finished TMA using Prefabricated recipient block technique (Kononen et al)

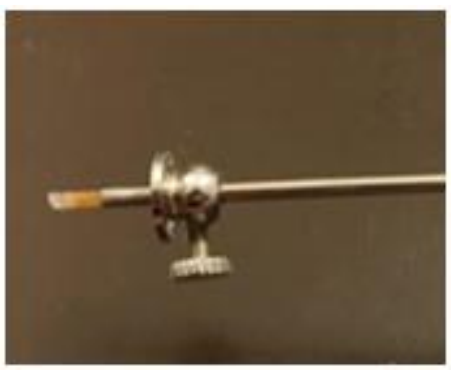

Figure F: Tissue core extracted for construction of TMA as suggested by Chen et al.

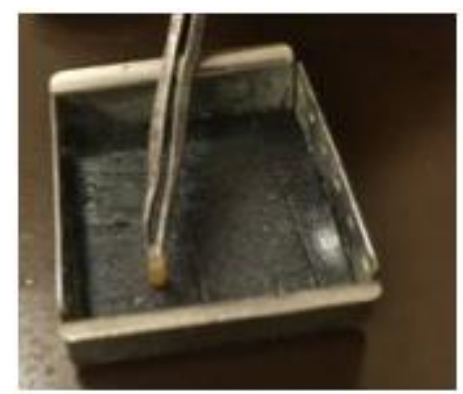

Figure G: Placement of tissue core on adhesive tape 


\section{JMSCR Vol||06||Issue||01||Page 31902-31914||January}

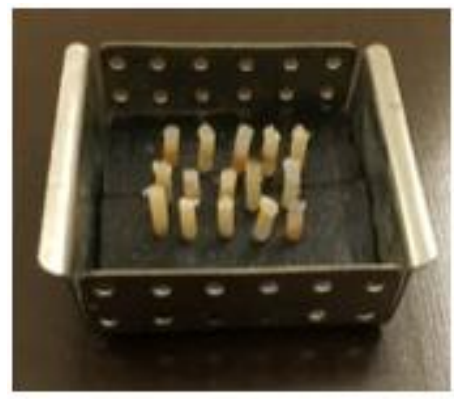

Figure H: 5 x 3 grid TMA constructed without prefabricated paraffin recipient block as suggested by Chen et al.

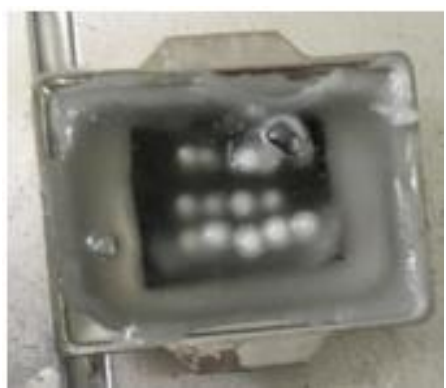

Figure I: Melted paraffin was being poured into TMA
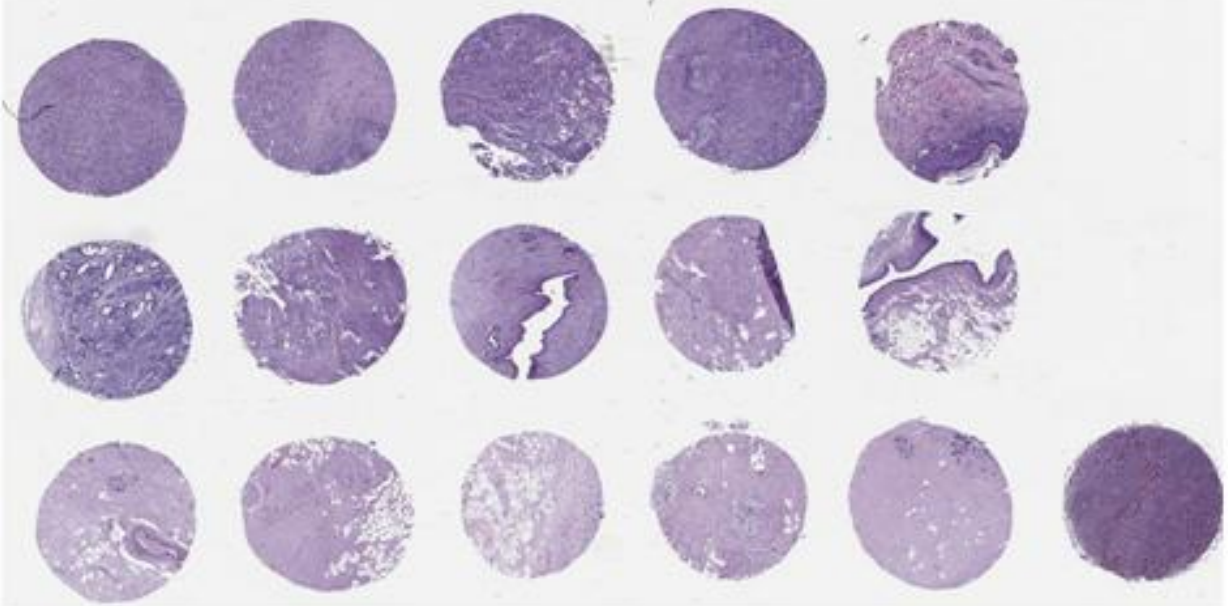

Figure J: Scanner view of H\&E section of TMA

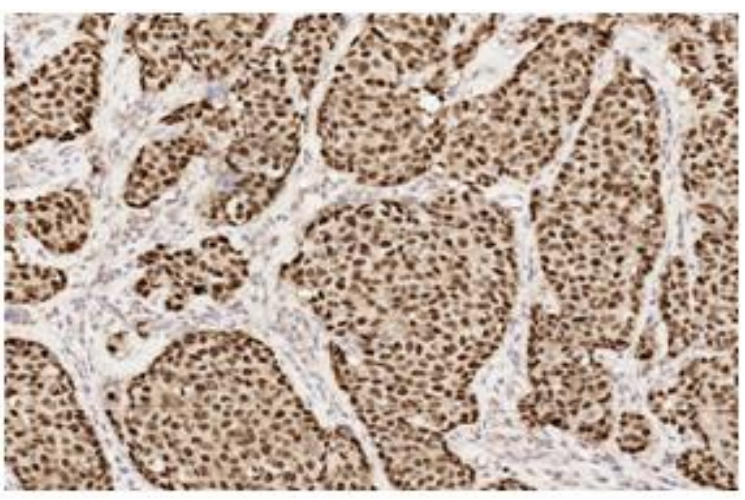

Figure L: 200X ER Positive Carcinoma Breast

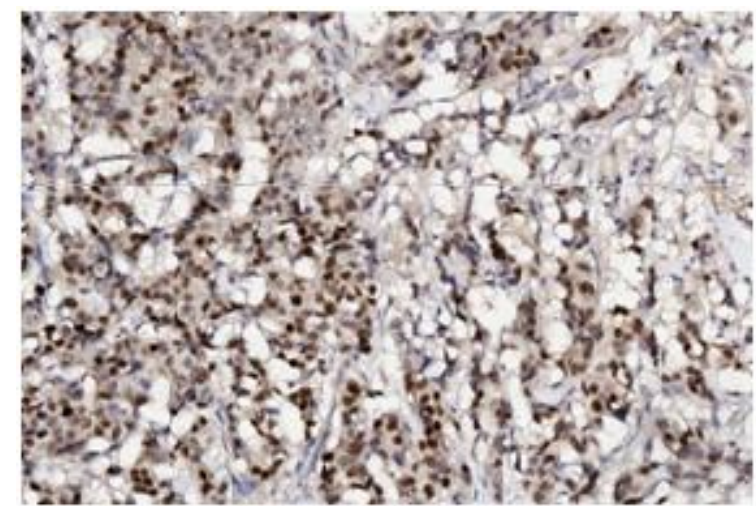

Figure K: 200X PR Positive Carcinoma Breast

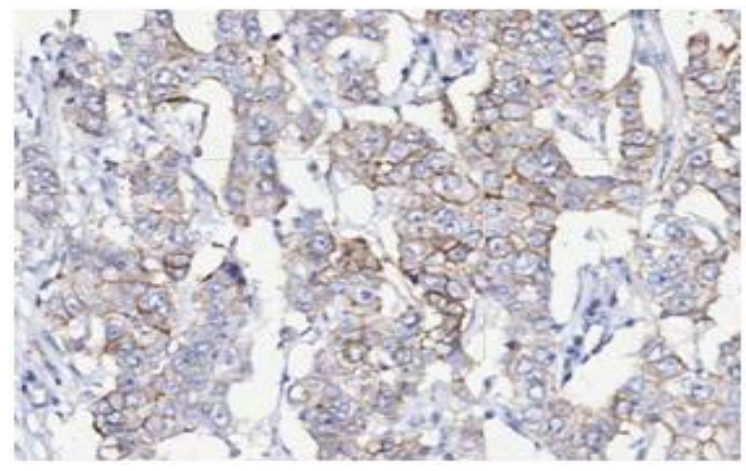

Figure M: 400X Her-2 Positive (2+) Carcinoma Breast

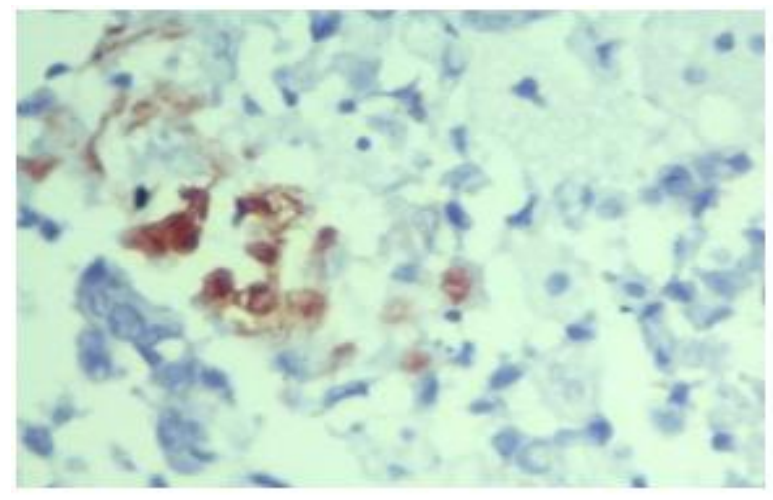

Figure N: 400X CK 5/6 Positive Carcinoma Breast 
Average Time Taken For Construction of Both Types Of TMA

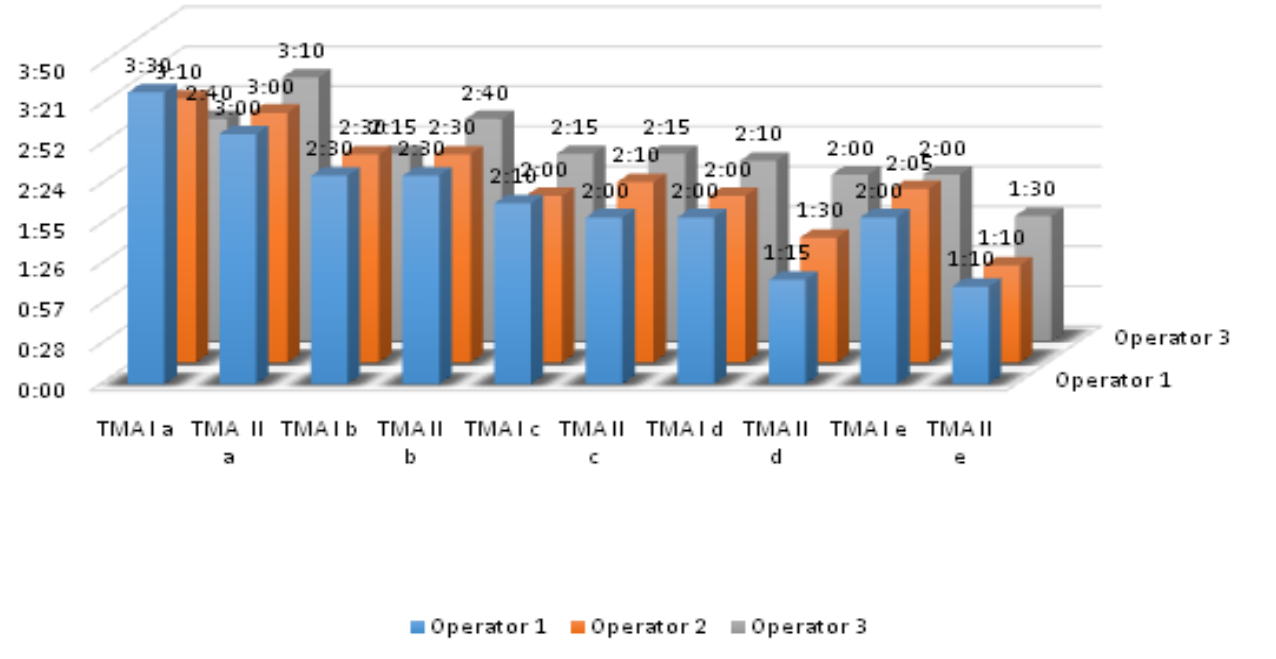

Figure O: Average Time Taken For Construction of both types of TMA

Number of sections in relation to cores in prefabricated recipient block method

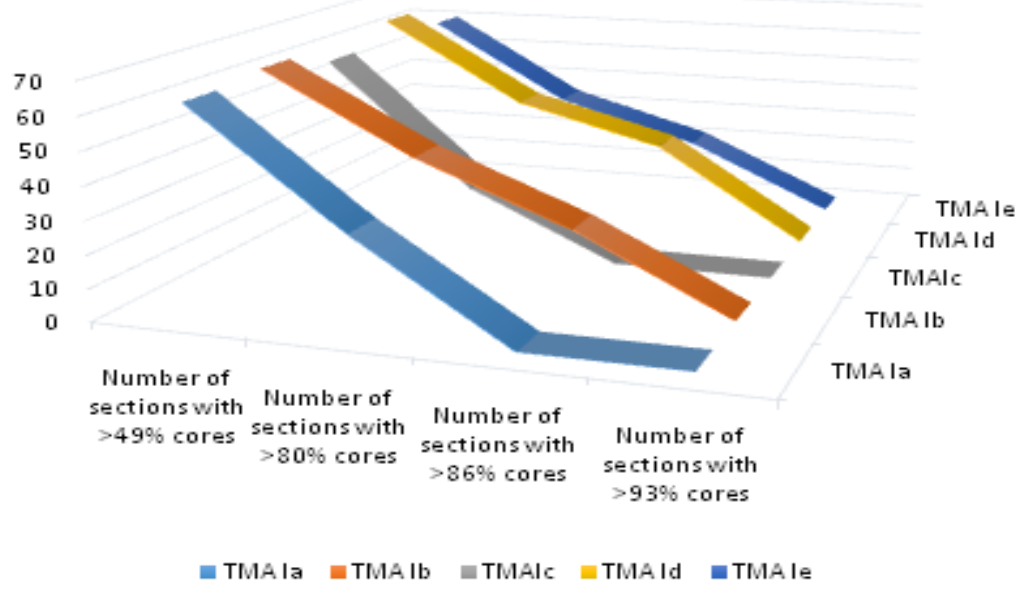

Figure P: Relation of number of section to cores in prefabricated recipient block method

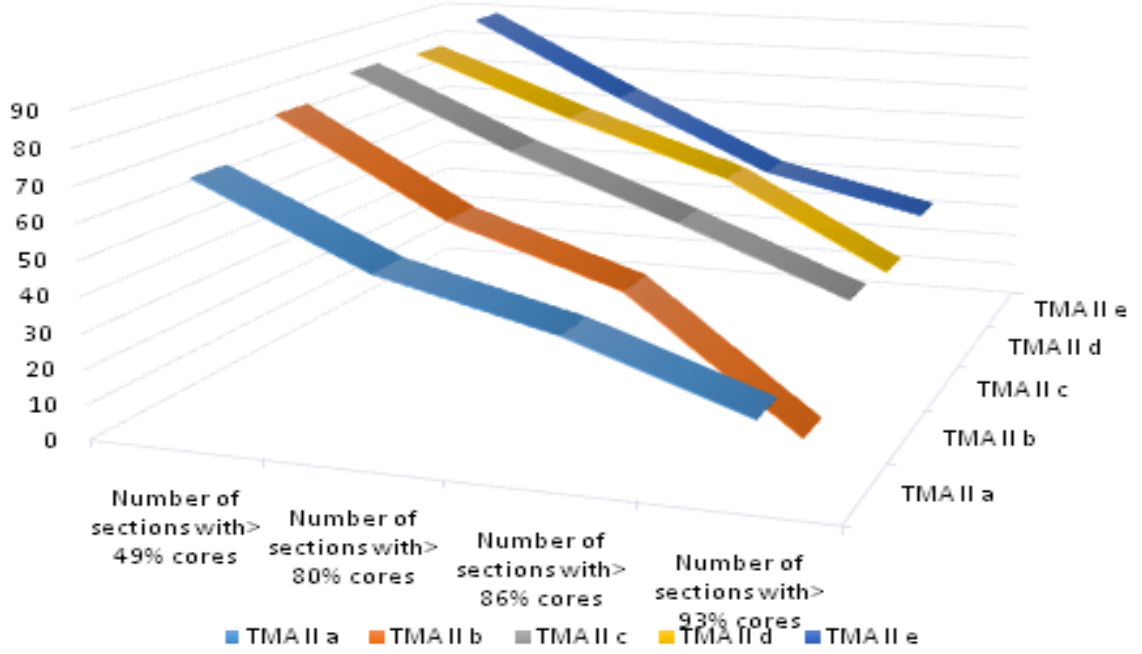

Figure Q: Relation of number of sections to cores in without prefabricated recipient block method 


\section{Discussion}

One of the major factors limiting the use of automated tissue microarray machines in India is the high cost of these machines. While the automated array machines from Beecher Instruments, San Prairie, Wisconsin, USA costs anywhere between 480,000 INR $(\$ 12,000)$ to $1,700,000 \operatorname{INR}(\$ 42,000)$ and even a manual tissue array system available from IHC World costs 48,000 INR (\$1200), including the recurring costs of the disposable parts and also maintainence. ${ }^{10}$

Manually constructing TMAs, such as in our case, the cost of the bone marrow biopsy needle set that was used was 400 INR (\$10), including the cost of getting the needles cut as per the requirement. It was comparable to the 1200 INR array set used by Singh et al. ${ }^{10}$ This difference in cost can be attributed to the different size needles that were used by them to obtain different core sizes. We used a single needle set of 14 and 16 gauge needles which gave a core size of $1.5 \mathrm{~mm}$. Other inexpensive standard stationary materials expended us an additional 200 INR.

The time taken to construct a microarray by both these methods was approximately 1 minute per core. So for a $5 \mathrm{X} 3$ grid, the average time taken was 15 minutes. This was comparable to the time taken by Singh et al. ${ }^{10}$ They took 10 minutes to construct a nine dot array and around 1 hour for a 56 dot array. Furthermore, the time taken to construct an array and stain one slide for immunohistochemical detection and $\mathrm{H} \& \mathrm{E}$ examination was markedly less (averagely 2hours 21 minutes for the first technique and about 2 hours 7 minutes averagely for the second technique, the difference between the both was statistically insignificant- $p$ value 0.074 ) than the time that would be taken to stain each tumor section separately for $\mathrm{H} \& \mathrm{E}$ and IHC examination.

\section{Problems Associated with the Technique Proposed by Kononen et al $^{\mathbf{1}}$}

There were certain problems that were encountered in the construction process by the first method. The recipient blocks can crack and break at the time of punching the hole. This is even more so in the winter months when the temperature is low. This problem can be decreased or avoided by slightly heating the blank wax blocks before starting the procedure. The slight heating makes the wax more moldable and hence prevents breakage. The recipient block was also more prone to cracking and paraffin bulging when the density of the cores were increased, this could however be prevented by maintaining adequate space between each hole punched (in our case we maintained it at least $1 \mathrm{~mm}$ apart), minimum recommended is $0.5 \mathrm{~mm} .{ }^{11}$ Another causative factor for block cracking was observed when cores were placed along the periphery of the block, thus we ensured that the cores were placed centrally leaving a margin of at least $2.5 \mathrm{~mm}$ on all sides.

A frequent problem observed by this method was presence of cores at different levels of depth in the recipient block. This was addressed by maintaining the punch length of standard size with the help of the bone marrow biopsy needle guard. Despite this measure this problem persisted, which in our opinion could be caused by tilting/ deviation of the needle while punching the hole in recipient block (which ideally should be perpendicular to the recipient block), which further led to mal-alignment of cores and also cores not being flushed to the surface (as seen significantly with this technique).

Another significant problem observed with this technique was broken cores while inserting them in the recipient block which may become significant when the donor block has limited tumor proper areas. Also, one key area which needed to be paid attention to was proper placement of donor cores in their specific punches in the recipient block. Once inserted, the retrieval of the core was extremely cumbersome (which could be attempted by inserting the biopsy needle back in the hole, and pulling it out in a to and fro twisting motion), which however led to enlargement of the recipient hole and also residual 
presence of the retrieved core, thus confounding results.

Any difference in the length of the hole that was punched out in the recipient hole and the length of the core tissue from the donor block could also cause difficulty in the donor core being flushed to surface.

\section{Problems Associated with the Technique Proposed by Chen et al $^{2}$}

The problems encountered in the second method were subjective difficulty reported by few operators in the placement of donor cores on the adhesive tape. This, in our opinion, could be addressed by practice.

If the donor block has scant tissue, then the merit of making TMA by this technique could be questionable since the cores require extra trimming in order to remove excess wax and also to ensure that the cores were vertically adhered to the double-sided tape as well as properly aligned. One minor problem associated inherently with this technique is the cumbersome removal of the double sided adhesive tape, which can be dealt with either using multiple strips of the adhesive tape on the same block or facing off the block (on the side of tape) with the microtome which may however lead to a loss of a few sections.

One significant problem seen only with this technique is core tipping. Averagely 1.2 cores tipped every 5X3 TMA prepared, while pouring wax, and further reorienting the cores post pouring the wax was extremely cumbersome. Pouring wax gently along the periphery of the mold prevented core tipping, however this required a level of expertise and proved to be a key step for the success of this technique.

\section{Objective Parameters}

The average time taken to construct TMA I was 2 hours 21 mins and TMA II was 2 hours 7 mins, though this difference was not statistically significant ( $p$ value 0.074 ).

With practice it was observed that time required for construction of each type of TMA by all three operators was reducing. However, the slope of fall, i.e. the trend of decreased time requirement with every practice was much higher in the Chen technique, hence proving to be an easier technique to learn.

In the Kononen technique about 3-4 cores were found mal-aligned in every 5X3 TMA prepared, reason being tilting/ deviation of the biopsy needle while punching holes into the recipient block. However, in the Chen technique about 2 cores averagely were found mal-aligned in every $5 \mathrm{X} 3$ TMA prepared, being attributed to the wax pouring step of this technique. This difference was statistically insignificant ( $\mathrm{p}$ value 0.066 ).

There were about 2-3 cores averagely missing in every 5X3 TMA prepared by the Kononen technique and about $0-1$ cores averagely missing in every 5X3 TMA prepared by Chen technique. This was mainly attributed to incomplete merging of the cores with surrounding paraffin, thus melting becomes a key step for the first technique in order to prevent this. This difference in our opinion is qualitatively significant, because loss of a core essentially is the loss of tumor details in a study, and thus defeats the purpose of simultaneously studying all cases. Cores with incomplete merging to the surrounding paraffin was seen only in an occasional section of TMA prepared by the Chen technique, this was secondary to trapped air bubbles while pouring wax into the mold. Studies done in the past have documented loss of core as a common problem to various techniques. $^{10,12}$

This according to them, could be reduced to a significant extent by the use of poly-lysinated slides for IHC. ${ }^{10}$ The loss of core during taking sections can be significantly reduced by properly cooling the block on ice and using fresh blades. ${ }^{10}$ All of the above measures were taken in our study. In addition, the TMA blocks should not be subjected to much trimming also. ${ }^{10}$

The loss of a single core becomes less significant for analytical purposes when multiple cores from the same tumor are taken. Also, in large case series, the loss of a few cores during processing 
and staining does not significantly alter the results. However, the loss of even a single core will become significant in our setup if this technology is used to determine the ER and HER2 status for breast cancer cases routinely.

Broken cores was a problem inherent to the first technique, averagely 1-2 cores were reported broken per 5X3 TMA prepared, which could be prevented with careful insertion and by using thicker cores 1.2-1.5 mm. Averagely 3-4 cores were not flushed to the surface by the Kononen technique and this problem again was inherent to it, due to either improper placement or different levels of recipient holes or varying lengths of donor cores and therefore resulted in loss of significant number of section until all cores were placed on the slide.

The average number of sections cut with more than $93 \%$ of cores in Kononen technique were 0.0 and by Chen technique 20.6. This difference is significant qualitatively because the Kononen technique hardly provided sections in which all the cores were present, which could entail loss of assessment of one or the other tumor specimen or too few slides if all the cores were to be studied together. On an average 20 sections were provided by the Chen technique with most of the cores, thus ensured that a battery of stains and procedures could be carried out on the sections.

Although the Chen technique ${ }^{2}$ can be used to construct TMAs with more than 300 tissue cores, it seemed (more comfortable in our experience), especially in research settings it is recommended that 30-50 tissue cores should be installed.

Therefore if few slides are necessary, fewer specimens (20-30) have to be studied and no precise arrangement is necessary then Kononen technique is recommended. And if, many slides are necessary, there are few specimens (30-50) and precise arrangement is necessary, then Chen technique: TMA with double sided adhesive tape is recommended. ${ }^{2,3}$

The Tissue microarray approach has been criticized for its use of small punches from tumors with an original size of up to several centimeters in diameter, comprising heterogenous areas of increased proliferation, apoptosis, matrix remodeling, necrosis, etc. ${ }^{13}$ For example, in breast cancer the grading is dependent on the presence and number of mitoses in the periphery of the tumor. Therefore, a core from the center of the tumor will not be an ideal core for grading. Also, a small core cannot represent the correct architectural pattern of the entire tumor which is also important for grading of breast carcinomas. Therefore, key consideration is the size of the punch. Four sizes are available: $0.6 \mathrm{~mm}, 1.0 \mathrm{~mm}$, $1.5 \mathrm{~mm}$, and $2.0 \mathrm{~mm}$. Although some investigators prefer the 2.0- $\mathrm{mm}$ size, these large cores may damage the donor and recipient blocks. If larger cores are desired, the $1.5-\mathrm{mm}$ core may be acceptable, as it seems to cause less damage but still covers a large area. The $1.5-\mathrm{mm}$ core is the preferred core size of most of the breast cancer arrays that have been constructed at our institution.

A different combination of needles used will be able to give a different core size which can be standardized by the laboratories as per the requirement. For making arrays to run controls for IHC, a smaller core size is usually sufficient. To study the patterns of expression of different markers in tumors and for research purposes, a larger core size may be required. For more heterogeneous tumors or lesions, it may be necessary to study an even larger area and a greater core size can be used. Taking multiple cores from the same tumor having different morphological patterns or grades can also reduce the chances of discrepancy between the entire tissue section and tissue microarray results for immunohistochemical analysis of tumors.

The use of tissue microarray sections significantly decrease the amount of time and reagents used for immunohistochemistry. Since we used a $5 \times 3$ grid, for 65 cases, five tissue microarray sections were processed. With the reagents and the time required for IHC analysis of 5 sections, the data for all the 65 cases could be assessed in a short span of time. As the entire cohort of cases being stained 
together are subjected to the exactly same processing, variations in staining due to changes in the $\mathrm{pH}$, temperature, staining time etc.

\section{Conclusion}

We found the Chen technique ${ }^{2}$ superior to the technique proposed by Kononen et al $^{1}$ in terms of number sections obtained with almost all cores, which is a significant factor when high throughput analysis of tumors is being done and a battery of procedures have to be done, defeating the purpose of simultaneously analyzing all specimen together.

\section{References}

1. Kononen J, Bubendorf L, Kallioniemi A, Bärlund M, Schraml P, Leighton S, et al. Tissue microarrays for high-throughput molecular profiling of tumor specimens. Nat Med. 1998;4:844-847.

2. Chen N, Zhou Q. Constructing tissue microarrays without prefabricating recipient blocks. A novel approach. Am. J. Clin. Pathol. 2005; 124: 103-107.

3. Wilkens L, Verfahren U. Vorrichtungzur Präparation von Gewebeproben. German Patent, DE.2003;524 A1:102-103.

4. Wang H, Zhang W, Fuller GN. Tissue microarrays: applications in neuropathology research, diagnosis, and education. Brain Pathology. 2002 Jan;12(1):95-107

5. Parkin DM, Bray FI, Devesa SS. Cancer burden in the year 2000. The global picture. European Journal of cancer. 2001;37: S4-S66.

6. Althuis MD, Dozier JM, Anderson WF, Devesa SS, Brinton LA. Global trends in breast cancer incidence and mortality 1973-1997. International Journal of Epidemiology. 2005;34:405-412.
7. Yeole BB. Trends in cancer incidence in female breast, cervix uteri, corpus uteri, and ovary in India. Asian Pacific Journal of Cancer Prevention.2008;9:119-122.

8. ICMR. National Cancer Registry programmme Population based cancer registries report. Two year report 2004-2005. Bangalore, India: ICMR December;2008.

9. Yeole BB, Kurkure AP. An epidemiological assessment of increasing incidence and trends in breast cancer in Mumbai and other cities in India, during the last two decades. Asian Pacific Journal of Cancer Prevention. 2003;4:51-56.

10. Singh DK, Sakhuja P, Gondal R. Making and Using inexpensive manually constructed tissue micro-array: Experience of a tertiary care hospital in India. Indian Journal Of Pathology and Microbiology. 2009;52(3):304-309.

11. Hoos A, Cordon-Cardo C. Tissue microarray profiling of cancer specimens and cell lines: Opportunities and limitations. Lab. Invest. 2001; 81: 1331-1338.

12. Parker RL, Huntsman DJ, Lesack DW, Cupples JB, Grant DR, Akbari M, et al. Assessment of Interlaboratory Variation in the Immunohistochemical Determinattion of Estrogen Receptor Status Using a Breast Cancer Tissue Microarray. American Journal of Clinical Pathology. 2002;117(5):723-728.

13. Packeisen J, Korsching E, Herbst $\mathrm{H}$, Boecker W, Buerger H. Demystified... Tissue microarray technology. Journal of Clinical Pathology. 2003;56:198-204. 